\title{
Susceptibility of Staphylococcus aureus Isolates Causing Bloodstream Infection to the Antimicrobial Peptide LL-37
}

\author{
Sinead McNicholas ${ }^{1,2}$, Hilary Humphreys ${ }^{1,2}$, Deirdre Fitzgerald Hughes ${ }^{\text {* }}$ \\ ${ }^{1}$ Department of Clinical Microbiology, RCSI Education and Research Centre, Beaumont Hospital, Dublin, Ireland \\ ${ }^{2}$ Department of Microbiology, Beaumont Hospital, Dublin, Ireland \\ Email: *dfitzgeraldhughes@rcsi.ie
}

Received January 2, 2013; revised February 8, 2013; accepted February 25, 2013

\begin{abstract}
Both bacterial and host factors contribute to complicated bloodstream infection (BSI) caused by Staphylococcus aureus including methicillin-resistant $S$. aureus (MRSA). One bacterial factor that may affect the persistence of $S$. aureus in complicated BSI is reduced susceptibility to the innate immune defence peptide LL-37. LL-37 susceptibility among $S$. aureus isolates causing uncomplicated and complicated BSI was investigated. Following incubation with $2.5 \mu \mathrm{g} / \mathrm{ml}$ LL-37 for $1 \mathrm{~h}$, the mean percentage survival was $67.6 \%$ and $74.9 \%$ for isolates causing uncomplicated and complicated BSI, respectively. Reduced LL-37 susceptibility may contribute to the persistence of $S$. aureus in complicated BSI.
\end{abstract}

Keywords: Staphylococcus aureus; Bloodstream Infection; LL-37

\section{Introduction}

The clinical outcome of bloodstream infection (BSI) caused by Staphylococcus aureus is variable and both bacterial and host innate immune factors may contribute to the clinical course of infection. The innate immune system acts as the first line of defence against microorganisms such as $S$. aureus and cationic anti-microbial peptides (CAMPs) are an important component of the innate immune response [1]. LL-37 is a cathelicidin, found in various cell types including neutrophils, monocytes, epithelial cells, salivary glands and sweat glands, and this molecule has important functions which include; the protection of skin and epithelial surfaces from invading organisms, the direct killing of phagocytosed bacteria and immune-regulation [1,2]. Its concentration on unstimulated mucosa ranges from $2-5 \mu \mathrm{g} / \mathrm{ml}$ but may increase in response to infection to levels above $30 \mu \mathrm{g} / \mathrm{ml}$ [3]. S. aureus has evolved mechanisms that confer reduced susceptibility to CAMPS such as LL-37, allowing it to evade this important component of innate immunity. Although these mechanisms are poorly understood, a number of potential factors have been identified and include aureolysin, which inactivates LL-37 and both polysaccharide intracellular adhesion (PIA) and fibrinogen-binding protein ( $\mathrm{fnBP}$ ) which have been shown to reduce susceptibility to LL-37 [4-6]. In addition, increased surface charge, achieved by the incorporation of D-alanine into teichoic acid or of lysine into phosphati-

\footnotetext{
"Corresponding author.
}

dylglycerol may reduce susceptibility to LL-37. Twenty $S$. aureus isolates causing BSI were assessed to determine if isolates differed in their susceptibility to LL-37 at physiological concentrations and whether in-vitro susceptibility to this peptide correlated with persistence or potential for complications of $S$. aureus BSI.

\section{Materials and Methods}

\subsection{Bacterial Strains and Growth Conditions}

Twenty $S$. aureus isolates that caused BSI in Beaumont Hospital Dublin between the years 2008-2011 were selected for this study. Ten isolates had caused uncomplicated BSI and 10 had caused a complicated infection, defined as a persistent (lasting $>3$ days despite appropriate antibiotics) or disseminated infection (e.g. osteomyelitis). Isolate details are summarized in Table 1. Three reference strains were also included, Methicillin-resistant $S$. aureus (MRSA) strain COL, and S. aureus SH 1000 (kind gifts from Dr. Timothy Foster, Trinity College Dublin and Dr. Simon Foster, University of Sheffield, UK) and S. aureus ATCC 44330.

\subsection{DNA Microarray and spa Typing}

A combination of DNA microarray and spa typing was used to determine the clonal complex (CC) to which each isolate belonged. DNA microarray was also used to determine the presence or absence of genes that may contribute to LL-37 susceptibility. Genomic DNA was 
Table 1. Details of $S$. aureus isolates from uncomplicated and complicated BSI, used for LL-37 bactericidal assay.

\begin{tabular}{|c|c|c|c|c|c|c|c|}
\hline Isolate No. & Organism & $m p r f^{a}$ & $a u r^{\mathrm{a}}$ & $i c a^{\mathrm{a}}$ & $f n B P A^{\mathrm{a}}$ & Capsule type $^{\mathrm{a}}$ & Clonal complex $(\mathrm{CC})^{\mathrm{b}}$ \\
\hline \multicolumn{8}{|c|}{ Uncomplicated } \\
\hline 11 & MRSA & + & + & + & + & 5 & $\mathrm{CC} 22$ \\
\hline 13 & MSSA & + & + & + & + & 8 & $\mathrm{CC} 15$ \\
\hline 16 & MSSA & + & + & + & + & 8 & $\mathrm{CC} 15$ \\
\hline 22 & MSSA & + & + & + & + & 5 & $\mathrm{CC} 25$ \\
\hline 23 & MSSA & + & + & + & + & 5 & $\mathrm{CC} 5$ \\
\hline 24 & MSSA & + & + & + & + & 5 & $\mathrm{CC} 22$ \\
\hline 30 & MSSA & - & + & + & + & 8 & $\mathrm{CC} 45$ \\
\hline 32 & MSSA & + & + & + & + & 8 & $\mathrm{CC} 15$ \\
\hline 50 & MSSA & + & + & + & + & 5 & $\mathrm{CC} 5$ \\
\hline 58 & MSSA & + & + & + & + & 8 & $\mathrm{CC} 30$ \\
\hline \multicolumn{8}{|l|}{ Complicated } \\
\hline 3 & MSSA & + & + & + & + & 8 & $\mathrm{CC} 12$ \\
\hline 7 & MRSA & + & + & + & + & 5 & $\mathrm{CC} 20$ \\
\hline 15 & MSSA & + & + & + & + & 8 & CC30 \\
\hline 17 & MSSA & + & + & + & + & 5 & $\mathrm{CC} 8$ \\
\hline 31 & MRSA & + & + & + & + & 5 & $\mathrm{CC} 22$ \\
\hline 33 & MSSA & - & + & + & + & 8 & $\mathrm{CC} 45$ \\
\hline 37 & MSSA & + & + & + & + & 8 & CC30 \\
\hline 48 & MSSA & + & + & + & + & 8 & $\mathrm{CC} 15$ \\
\hline 49 & MRSA & + & + & + & + & 5 & $\mathrm{CC} 22$ \\
\hline 53 & MSSA & + & + & + & + & 5 & $\mathrm{CC} 5$ \\
\hline
\end{tabular}

${ }^{\mathrm{a}}$ Presence or absence of gene determined by DNA microarray; ${ }^{\mathrm{b}}$ Clonal lineage determined by a combination of DNA microarray and spa typing as described in the methods. Mprf, encodes multiple peptide resistance factor, aur encodes aureolysin, ica encodes intracellular adhesion, fnBPA encodes fibrinogen binding protein.

extracted using a DNeasy ${ }^{\circledR}$ blood and tissue kit (Qiagen, Crawley, UK). Spa typing, which involves PCR amplification and sequencing of the polymorphic 24 base pair variable number tandem repeat region within the 3 ' end of the protein A gene spa, was carried out according to the SeqNet website (http://www.seqnet.org). Sequencing was performed by Beckman Coulter Genomics (Takeley, UK) and Source BioScience (Dublin, Ireland). Genetic characterization of isolates was undertaken using the StaphyType Kit (Alere Technologies Germany) as previously described [7].

\subsection{LL-37 Bactericidal Assay}

A modification of the method described by Ouhara et al. (2008) [8] was used. Briefly, colony survival was determined from viable cell counts following incubation of 5 $\times 10^{5} \mathrm{CFU} / \mathrm{ml}$ S. aureus with $2.5 \mu \mathrm{g} / \mathrm{ml}$ LL-37 in $10 \mathrm{mM}$ sodium phosphate buffer, $\mathrm{pH} 7.4$ for $1 \mathrm{~h}$ and dilution $(1 / 10)$ with $0.95 \% \mathrm{w} / \mathrm{v} \mathrm{NaCl}$. Percentage survival was calculated from viable counts $(\mathrm{CFU} / \mathrm{ml})$ from assays containing LL-37 compared to control assays containing no LL-37. The assay was performed in duplicate on three occasions for each isolate and the reference strains. Preliminary investigation of laboratory strains of $S$. aureus demonstrated concentration-dependent susceptibility to LL-37. The lowest concentration tested $(2.5 \mu \mathrm{g} / \mathrm{ml})$ allowed the observation of susceptibility differentials between strains $(24 \%, 54 \%, 92 \%$ survival for $\mathrm{COL}, \mathrm{SH}$ 1000 and 8325-4 respectively) and this concentration was therefore chosen for investigation of clinical isolates of $S$. aureus.

\subsection{Statistical Analysis}

Mean survival following incubation with LL-37 was 
compared between isolates from uncomplicated $V s$ complicated infection using the Student's t-test for statistical significance.

\section{Results}

\subsection{Survival of $S$. aureus Following Incubation with LL-37}

Among $S$. aureus clinical isolates causing complicated and uncomplicated BSI there was a wide variation in susceptibility to killing by LL-37 under the assay conditions used (uncomplicated BSI isolates, \% survival; range $=24.2 \%-100 \%$, mean $=67.6 \%$, complicated $S$. aureus BSI isolates, $\%$ survival; range $=52.8 \%-100 \%$, mean $=$ $74.9 \%$. However, overall, $S$. aureus isolates causing uncomplicated BSI were more susceptible to killing by LL37 than isolates causing complicated BSI but this difference was not statistically significant ( $p=0.48$, Student's t-test) (Figure 1(a)). MRSA isolates were more resistant to LL-37 killing (percentage survival, range 57.94\% $100 \%$, mean $80.5 \%$ ) than MSSA isolates (percentage survival, range $24.2 \%-100 \%$, mean $68.9 \%$ ) but this difference was not statistically significant $(p=0.47)$ (Figure 1(b)).

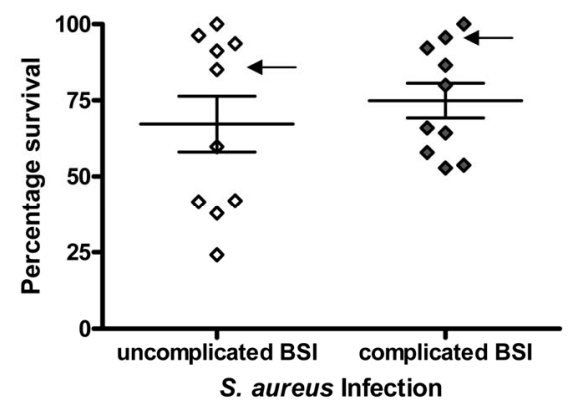

(a)

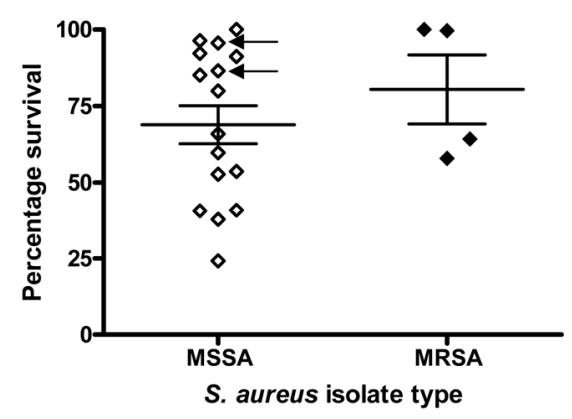

(b)

Figure 1. LL-37 Susceptibility of $S$. aureus isolates causing BSI. The percentage survival after exposure to $2.5 \mu \mathrm{g} / \mathrm{ml} \mathrm{LL}-$ 37 for $1 \mathrm{~h}$ is shown. Each symbol represents \% survival calculated from assays carried out in duplicate on three separate occasions (a) $S$. aureus isolates causing uncomplicated BSI $(n=10)$ and complicated BSI $(n=10)$; (b) MSSA isolates causing BSI $(n=16)$ and MRSA isolates causing BSI $(n=4)$. Arrows indicate the $S$. aureus isolates that were mprf.

\subsection{LL-37 Susceptibility, Clonal Complex and Carriage of Selected Virulence Genes}

$S$. aureus isolates causing both complicated and uncomplicated infections belonged to a variety of CCs (Table 1) but only CCs $5,15,22,30$, and 45 were common to isolates from complicated and uncomplicated infections. The LL-37 susceptibility of the most prevalent CCs (CC15(4), CC22(4), CC5(3) CC30(3)), were compared but there was no statistically significant difference in LL-37 bactericidal activity against isolates from different CCs (data not shown).

Genes that encode virulence factors that may influence LL-37 susceptibility among $S$. aureus, include mprf, aur, ica and $f B P P$. Microarray analysis revealed the carriage of mprf in 18/20 S. aureus isolates (9/10 complicated and $9 / 10$ uncomplicated) and the other three genes were found in all isolates investigated (Table 1). LL-37 susceptibility of the two mprf isolates was comparable to a number of mprf isolates. The two mprf isolates are highlighted in Figure 1. Genes encoding capsule types 3 and 8 were equally distributed among isolates from complicated and uncomplicated infections (Table 1). Furthermore, no statistically significant difference in LL-37 susceptibility between capsule type 5 and capsule type 8 $S$. aureus isolates was found $\left(74.4 \% V_{S} 68.1 \%\right.$ survival respectively, $p=0.74$ ).

\section{Discussion}

There was marked variation in the in-vitro susceptibility of $S$. aureus isolates to LL-37 and high variation has been previously reported among other collections of $S$. aureus clinical isolates [9]. Cell survival following incubation with LL-37 was greater for $S$. aureus isolates causing complicated BSI compared to uncomplicated BSI. Although this is the first study that we are aware of, that assessed bloodstream isolates, the numbers studied may have precluded the demonstration of statistical significance. The relatively low rates of complicated cases of BSI caused by $S$. aureus (less than $15 \%$ of $S$. aureus BSI in our hospital during the study period) was a limiting factor in the present study. Therefore further studies are required on larger numbers of isolates from more than one centre.

It has previously been shown that MRSA isolates are more susceptible to killing by LL-37 than MSSA isolates $[5,8,10]$. Although the number of isolates studied here were relatively low, this pattern of MRSA/MSSA comparative susceptibility was not found in $S$. aureus causing BSI. Removing the four MRSA isolates from the previous comparison of uncomplicated $V S$ complicated infections did not result in a significant change in the statistical comparison of LL-37 susceptibility (percentage survival $64.1 \% V_{S} 75.3 \%, p=0.39$ ). 
LL-37 has been shown to play an important role in the innate immune response to $S$. aureus infection However, $S$. aureus has evolved mechanisms that confer reduced susceptibility to LL-37 (e.g. increasing surface charge, inactivation of LL-37 by aureolysin) [4-6,10,11]. A number of other factors (PIA production, fnbP and the presence of a capsule) have also been shown to influence susceptibility of $S$. aureus isolates to killing by CAMPs, including LL-37 although the exact mechanisms are unknown [4,5]. The ability of certain $S$. aureus isolates to evade and inactivate this important antimicrobial peptide may contribute to their persistence in complicated BSI. However resistance to $\beta$-lactam antibiotics, capsule type or clonal type of the isolate did not significantly influence in-vitro susceptibility to LL-37.

Variations in LL-37 susceptibility among the isolates were also independent of the presence of genes encoding lysylphosphatidylglycerol synthetase (mprf), polysaccharide intracellular adhesin (ica), fibronectin binding protein $(f n b A B)$ and aureolysin (aur) as all isolates investigated, contained them (except two which did not contain $m p r f$ ). S. aureus causing BSI may produce other as yet unidentified virulence factors that may protect against innate immune defences. It is also possible that the expression of these genes varies among $S$. aureus isolates, which may contribute to the variations found. Expression of virulence genes is highly dependent on the dynamic environment encountered by the organism during infection and the killing assay used here does not facilitate investigation of the effects of these factors on LL-37 susceptibility.

In conclusion, LL-37 susceptibility may play a role in the persistence or potential for complications of $S$. aureus BSI, but the complex roles of LL-37 in the immune response against $S$. aureus and the significance of LL-37 resistance among $S$. aureus isolates, warrants further study.

\section{Acknowledgements}

We are grateful to the staff of the Microbiology Department, Beaumont Hospital, who provided S. aureus isolates. The authors acknowledge financial support from Pfizer Ireland through an Educational Award, No. WS 376235.

\section{REFERENCES}

[1] J. D. Heilborn, M. F. Nilsson, G. Kratz, G. Weber, O. Sorensen, N. Borregaard and M. Stahle-Backdahl, "The Cathelicidin Anti-Microbial Peptide LL-37 Is Involved in Re-Epithelialization of Human Skin Wounds and Is Lacking in Chronic Ulcer Epithelium," Journal of Investigative Dermatology, Vol. 120, No. 3, 2003, pp. 379-389. doi:10.1046/j.1523-1747.2003.12069.x
[2] A. R. Koczulla and R. Bals, "Antimicrobial Peptides: Current Status and Therapeutic Potential," Drugs, Vol. 63, No. 4, 2003, pp. 389-406. doi:10.2165/00003495-200363040-00005

[3] J. Overhage, A. Campisano, M. Bains, E. C. Torfs, B. H. Rehm and R. E. Hancock, "Human Host Defense Peptide LL-37 Prevents Bacterial Biofilm Formation," Infection and Immunity, Vol. 76, No. 9, 2008, pp. 4176-4182. doi:10.1128/IAI.00318-08

[4] I. Fedtke, F. Gotz and A. Peschel, "Bacterial Evasion of Innate Host Defenses-The Staphylococcus aureus Lesson," International Journal of Medical Microbiology, Vol. 294, No. 2-3, 2004, pp. 189-194. doi:10.1016/j.ijmm.2004.06.016

[5] H. Komatsuzawa, K. Ouhara, S. Yamada, T. Fujiwara, K. Sayama, K. Hashimoto and M. Sugai, "Innate Defences against Methicillin-Resistant Staphylococcus aureus (MRSA) Infection," Journal of Pathology, Vol. 208, No. 2, 2006, pp. 249-260. doi:10.1002/path.1898

[6] M. Sieprawska-Lupa, P. Mydel, K. Krawczyk, K. Wojcik, M. Puklo, B. Lupa, P. Suder, J. Silberring, M. Reed, J. Pohl, W. Shafer, F. McAleese, T. Foster, J. Travis and J. Potempa, "Degradation of Human Antimicrobial Peptide LL-37 by Staphylococcus aureus-Derived Proteinases," Antimicrobial Agents and Chemotherapy, Vol. 48, No. 12, 2004, pp. 4673-4679. doi:10.1128/AAC.48.12.4673-4679.2004

[7] S. McNicholas, A. C. Shore, D. C. Coleman, H. Humphreys and D. F. Hughes, "DNA Microarray Genotyping and Virulence and Antimicrobial Resistance Gene Profiling of Methicillin-Resistant Staphylococcus aureus Bloodstream Isolates from Renal Patients," Journal of Clinical Microbiology, Vol. 49, No. 12, 2011, pp. 4349-4351. doi:10.1128/JCM.05017-11

[8] K. Ouhara, H. Komatsuzawa, T. Kawai, H. Nishi, T. Fujiwara, Y. Fujiue, M. Kuwabara, K. Sayama, K. Hashimoto and M. Sugai, "Increased Resistance to Cationic Antimicrobial Peptide LL-37 in Methicillin-Resistant Strains of Staphylococcus aureus," Journal of Antimicrobial Chemotherapy, Vol. 61, No. 6, 2008, pp. 1266-1269. doi:10.1093/jac/dkn106

[9] K. Midorikawa, K. Ouhara, H. Komatsuzawa, T. Kawai, S. Yamada, T. Fujiwara, K. Yamazaki, K. Sayama, M. A. Taubman, H. Kurihara, K. Hashimoto and M. Sugai, "Staphylococcus aureus Susceptibility to Innate Antimicrobial Peptides, Beta-Defensins and CAP18, Expressed by Human Keratinocytes," Infection and Immunity, Vol. 71, No. 7, 2003, pp. 3730-3739. doi:10.1128/IAI.71.7.3730-3739.2003

[10] T. J. Foster, "Immune Evasion by Staphylococci," Nature Reviews Microbiology, Vol. 3, No. 12, 2005, pp. 948-958. doi:10.1038/nrmicro1289

[11] S. H. Rooijakkers, K. P. van Kessel and J. A. van Strijp, "Staphylococcal Innate Immune Evasion," Trends in Microbiology, Vol. 13, No. 12, 2005, pp. 596-601. doi:10.1016/j.tim.2005.10.002 